\title{
Brief review of beneficial properties of aquatic exercise
}

\begin{abstract}
Although the benefits of aquatic exercise have been known for hundreds if not thousands of years only recently have the benefits and physiological responses to aquatic exercise have been studied. Immersion in water has a number of beneficial effects on the human body which can be utilised for many populations for rehabilitation, remedial exercise, recovery and well beginning.
\end{abstract}

Keywords: buoyancy, immersion, rehab, aquatic, hydro
Volume 7 Issue 4 - 2017

\author{
Stephen John Payton \\ Department of Sport and exercise, Teesside University, UK
}

Correspondence: Stephen John Payton, Department of Sport and exercise, Teesside University, Middlesbrough, Tees Valley, TSI 3BX, UK, Email s.payton@tees.ac.uk

Received: April 08, 2017| Published: June 09, 2017

\section{Introduction}

There is an increase in the number of people taking part in water based activities whether it is healthy population or disability population or for specific rehabilitation. Exercise in water can be modified to suit the age and fitness levels. ${ }^{1}$ There is some wellestablished physiological responses to exercise in water such as reduced impact on joints decrease impact on joints, ${ }^{2}$ There is a growing understanding of the physiological responses to exercise in water compared to land based exercise such as changes in blood flow and water temperature. ${ }^{3-5}$ An increase in Range of motion, ${ }^{6}$ and heart rate response due to immersion. ${ }^{7}$ Reduced delayed onset muscle soreness post exercise. ${ }^{8}$ This short review will highlight some of the key benefits of aquatic exercise.

\section{Hydro static pressure}

Water is 829times denser than air at 30degrees. Water is exerts pressure which is proportional $1 \mathrm{~mm} \mathrm{Hg}$ per $1.36 \mathrm{~cm}$ in depth at $121 \mathrm{~cm}$ the pressure is at $88.9 \mathrm{mmHg}$ which is higher than normal diastolic blood pressure of $80 \mathrm{~mm} \mathrm{Hg}$ this pressure at a depth of $121 \mathrm{~cm}$ facilitates blood back to the heart. This increase in pressure (hydrostatic pressure) surrounding the body has been identified to have the effect in a drop in the participants resting heart rate. Hydrostatic pressure facilitating venous return can be used for the benefit populations who have low exercise tolerance enabling them to exercise for longer.

\section{Buoyancy}

The force for of gravity acts upon the human body on every activity on land. When immersed in water the body is subjected to opposing force "buoyancy" this force acts apposing direction to gravity the greater the depth of water the body is immersed in the greater the force of buoyancy. This buoyancy of can facilitate movement in an upward direction as long as the movement is slow. The effect of reducing loading on the human body an increase in depth of water there is a decrease in impact on the musculoskeletal system submersion at immersion to the pubis symphysis results in an offloaded of 50. This up thrust from buoyancy and resistance in downward plane reduces the impact of landing and ultimately reduces eccentric load. Water can be used to facilitate learning movement patterns either post-surgery or for disability populations, water provide greater kinaesthetic awareness of movements this can increased feedback to the patient can help them be more aware of the positioning of their body at any one time and ultimately help rehabilitate the required movement pattern.

\section{Density and S\&C}

Another property of water is its density which can be utilized for strength and conditioning. As the body is surrounded by water it makes it possible to mimic sport specific movements and can be utilized from early stage rehabilitation as the water facilitates the movement to late stage. During late stage rehabilitation the water can be used as a resistance tool. The speed of the movement through water really distinguishes whether the water is a facilitator of movement or acts as a resistance tool. The change from facilitator to resistance occurs at speeds above 90 degrees per second. When performing say a vertical jump in the water the surrounding body of water acts as a resistance resulting in greater concentric force. A limb moving through the water is subject to drag force under turbulent conditions this resistance increases as a log of the velocity, so doubling the speed of movement to say 180 degrees requires eight times the power. Turbulent conditions are created moving through water faster and or through cutting the surface of water. The great aspect of creating force in the water is as soon as you stop moving say when a patient feels pain the force drops immediately this provides a safe environment to rehabilitate an athlete. Resistance drops to zero almost immediately on cessation of force because there is only a small amount of inertial moment as viscosity effectively counteracts inertial momentum. ${ }^{9}$ Technique is a major focus of plyometric exercise in rehabilitation is assisting the athlete with skill reacquisition and the establishment of biomechanically safe technique that will allow the athletes to achieve optimal performance.

\section{Thermal effect}

Water has a heat capacity 1000times greater than air and is 25 times faster at conducting heat than air. This allows water to be great medium for cooling down or heating up the soft tissue. This phenomenon is widely used to great affect post injury with the application of cold water immersion to aid recovery. Reduces muscle damage and discomfort, possibly contributing to a faster recovery of neuromuscular function. ${ }^{10}$

The ability to transfer heat much quicker and maintain the immersed body warm helps reduce the pain the client. When rehabilitating athletes pain can be a limiting factor to progress onto functional rehabilitation. Pain is the response to injury, nociceptors are activated they transmit nerve signals that travel through the spinal cord to the brain, where the sensation of pain is recognized. At the same time, neurotransmitters initiate a spinal reflex that increases muscle motor activity and tonicity at the site of injury, leading to a reflexive muscle 
contraction this result in a pain. To break this cycle heat can be applied Thermo receptors, special temperature-sensitive nerve endings, are activated by changes in skin temperature. These receptors initiate nerve signals that block nociception (the pain signal processing those results from a noxious stimulus) and activate thermo receptors theses block the pain signals. Heat transfer is 25 times more efficient in water than air which will help suppress pain the athlete is complaining off and lead to the patient performing there remedial exercise, ${ }^{11}$ participants found it more easily perform muscle exercises first in an aquatic environment before moving to land based rehabilitation. This research suggesting that the therapeutic pool is a safe environment for the exercise due to the reduction of pain threshold being suppressed in water. ${ }^{12}$ With the pain cycle being suppressed remedial exercise can then be performed which can then ultimately lead to rehabilitation transferring to the terrestrial environment.

\section{Range of motion}

An often overlooked benefit from being submerged in the water is that water acts as a supportive environment. The combination of viscosity of water and buoyancy can be used to aid movement and increase range of motion.. Performing exercise in water provides far move feedback to the athlete on body position to help perfect correct technique. This has the positive effect of helping to facilitate range of movement while performing slow movements. This can result in the athlete performing movements through a larger ROM than on land. Furthermore as the water surrounds the athlete, the athlete can perform a huge variety of functional sport specific movements. This positive effect has been reported by. ${ }^{13-15}$ Furthermore the heat transfer effect of the warm water will increase soft tissue temperature resulting in vasodilation and elongation of the tissue and an increase in core body temperature. ${ }^{16}$

\section{Conclusion}

There is evidence to support aquatic exercise for a wide range of conditions and populations. Further studies are required to provide a greater base of support to utilize the aquatic environment to its full potential.

\section{Acknowledgments}

None.

\section{Conflicts of interest}

Author declares there are no conflicts of interest.

\section{Funding}

None.

\section{References}

1. Yazigi F, Pinto S, Colado J, et al. The cadence and water temperature effect on physiological responses during water cycling. Eur J Sport Sci. 2013;13(6):659-665.
2. Robinson LE, Devor ST, Merrick MA, et al. The effects of land vs. aquatic plyometrics on power, torque, velocity, and muscle soreness in women. J Strength Cond Res. 2004;18(1):84-91.

3. Becker BE, Hildenbrand K, Whitcomb RK, et al. Biophysiologic effects of warm water immersion. International Journal of Aquatic Research and Education. 2009;3(1):24-37.

4. Castro Snchez AM, Matarn-Peñarrocha GA, Lara Palomo I, et al Hydrotherapy for the treatment of pain in people with multiple sclerosis: a randomized controlled trial. Evid Based Complement Alternat Med. 2011;2012:473963.

5. Tomas Carus P, Häkkinen A, Gusi N, et al. Aquatic training and detraining on fitness and quality of life in fibromyalgia. Med Sci Sports Exerc. 2007;39(7):1044-1450.

6. Irandoust $\mathrm{K}$, Taheri $\mathrm{M}$. The effects of aquatic exercise on body composition and nonspecific low back pain in elderly males. J Phys Ther Sci. 2006;27(2):433-435.|

7. Shono T, Fujishima K, Hotta N, et al. Cardiorespiratory response to low-intensity walking in water and on land in elderly women Journal of physiological anthropology and applied human science. 2001;20(5):269-274.

8. Donoghue OA, Shimojo H, Takagi H. Impact forces of plyometric exercises performed on land and in water. Sports Health. 2011;3(3):303-309.

9. Pöyhönen T, Keskinen KL, Kyröläinen H, et al. Neuromuscular function during therapeutic knee exercise under water and on dry land Arch Phys Med Rehabil. 2001;82(10):1446-1452.

10. Ascensão A, Leite M, Rebelo AN, et al. Effects of cold water immersion on the recovery of physical performance and muscle damage following a one-off soccer match. J Sports Sci . 2011;29(3):217-225.

11. Denning WM, Bressel E, Dolny D, et al. A review of biophysical differences between aquatic and land-based exercise. International Journal of Aquatic Research and Education. 2012;6(1):7.

12. Becker BE. Aquatic therapy: scientific foundations and clinical rehabilitation applications. PM R. 2009;1(9):859-872.

13. Barbosa TM, Marinho DA, Reis VM, et al. Physiological assessment of head-out aquatic exercises in healthy subjects: a qualitative review. $J$ Sports Sci Med. 2009;8(2):179-189.

14. Ruoti RG, Morris DM, Cole AJ. Aquatic rehabilitation Lippincott Williams \& Wilkins. 1997.

15. Pöyhönen T, Kyröläinen H, Keskinen KL, et al. Electromyographic and kinematic analysis of therapeutic knee exercises under water. Clin Biomech (Bristol, Avon). 2001;16(6):496-504.

16. Bleakley CM, Costello JT. Do thermal agents affect range of movement and mechanical properties in soft tissues? A systematic review. Arch Phys Med Rehabil. 2013;94(1):149-163. 